\title{
Ethnologische Feldforschung in Papua New Guinea
}

\author{
Alfred Bühler zum 80. Geburtstag
}

Das Ethnologische Seminar der Universität Basel hat in den Jahren 1972-74, unterstützt vom Schweizerischen Nationalfonds, ein größeres Projekt völkerkundlicher Feldarbeit im mittleren Sepik-Gebiet des nördlichen Neuguinea durchgeführt. Über diese Expedition, die hier nur als ein Beispiel für viele ähnliche gewählt wurde, soll im folgenden berichtet werden - jedoch weniger im Hinblick auf ihre wissenschaftlichen Einzelergebnisse, die bis jetzt den Umfang mehrerer Bände ${ }^{1)}$ erreicht haben, als vielmehr hinsichtlich der allgemeineren Überlegungen, die man heute - naturgemäß mit wechselndem Gewicht - in der Ethnologie zur Planung, Durchführung und Auswertung solcher Forschungsreisen anstellt, und der Probleme, die sich dabei ergeben.

Seit dem Erwachen des wissenschaftlichen Interesses an den Kulturen fremder Völker war die im fremden Kulturraum stattfindende völkerkundliche Primärforschung, in der Ethnologie als «Feldarbeit» oder "Feldforschung» bezeichnet und weithin, aber nicht ganz zutreffend mit «Ethnographie» gleichgesetzt, im wesentlichen auf drei Erkenntnisziele bezogen, die von den einzelnen Forschern bzw. innerhalb der verschiedenen wissenschaftlichen und geistesgeschichtlichen Richtungen im Laufe der Jahrtausende mit unterschiedlicher Akzentuierung verwirklicht wurden; der direkte Einbau fremder, also nicht selbst vor Ort erhaltener Mitteilungen in die eigene Darstellung, soweit sie eine vornehmlich beschreibende blieb, sei bei diesem Überblick zunächst miteingeschlossen.

Eine erste Forschungsmotivation läßt sich beschreiben als das im guten Sinn neugierige Interesse an der Vielfalt der Lebensformen in anderen Weltgegenden; dieses Wissenwollen ist durchwegs großräumig und additiv angelegt, greift von einer Region in die nächste hinüber und berichtet immer Neues, jedoch meistens Partikulares und ist auf das von der eigenen kulturellen Beschaffenheit oder von dem gerade neu Gesehenen wiederum Abweichende konzentriert, weil eben nur dieses in den Blick tritt. Als Beispiel dafür sei auf HERODOT ${ }^{2)}$ oder die frühen Berichte derjenigen Entdeckungsreisenden verwiesen, die die Kontinente durchquerten und denen es schon die äußeren Umstände verboten, sich jahrelang in einem einzigen neuen $\mathrm{Kul}$ - turgebiet aufzuhalten; der dadurch unvermeidliche Mangel an Vollständigkeit der Kulturbeschreibung wird jedoch häufig wettgemacht durch die Originalität der Anschauung und den vergleichenden Blick, der nun einmal auf Möglichkeiten zum Vergleich angewiesen ist.

Das zweite Erkenntnisziel wird gerade von der umgekehrten Interessenrichtung geprägt: statt großräumig ist es kleinräumig, statt additiv integrativ, und an die Stelle des erlebenden und erfahrenden Durchschreitens immer neuer Regionen tritt die Versenkung in eine einzige Kultur (nach dem jeweiligen historischen Maßstab) mit dem Ziel, hier möglichst viel zu wissen, auch die Details nicht zu übersehen und vor allem in einem zweiten Forschungsschritt den innerkulturlichen Zusammenhang des zunächst einzeln Erkannten zu erfassen. Als Beispiele für diesen "monographischen» Forschungsansatz könnten aus der Wissenschaftsgeschichte der Ethnologie etwa die Germanenbeschreibung des TACITUS ${ }^{3)}$ oder die Aztekentexte des FRAY BERNARDINO DE SAHAGÚN ${ }^{4}$ ) genannt werden. Steht hierbei die Aufschlüsselung des Fremden im Vordergrund, die ja nie anders denkbar ist denn als eine Art bilateraler Prozeß mit stetem - explizitem oder implizitem - Rückbezug auf das eigene Begriffsystem und infolgedessen mit gelegentlich deutlicherem Vergleich mit den eigenen kulturellen Normen, so führt die dritte Hauptvariante völkerkundlichen Erkenntnisstrebens über diese Konzentration auf eine einzelne Fremdkultur hinaus und sucht an ihrem Beispiel menschlich-kulturell Prinzipielles zu erfassen, Gesetzmäßigkeiten zu beschreiben oder durch eine umfassendere historische Perspektive das Einzelne in den größeren Zusammenhang zu stellen. Weil ein solcher, schon in stärkerem Maße theoretischer Anspruch im allgemeinen nur auf der Basis von breiterem Datenmaterial zu erfüllen ist, als im Rahmen einer einzelnen Feldarbeit beschafft werden kann, und deshalb normalerweise auch das am Schreibtisch stattfindende Literaturstudium einschließt, wird hierbei die Grenze von der «Ethnographie» zur «Ethnologie» - wie immer man die beiden Begriffe voneinander abhe-

Prof.Dr. Meinhard Schuster, Ethnologisches Seminar der Universität Basel, Münsterplatz 19, 4051 Basel. 
ben mag - deutlich überschritten und damit auch der engere Bereich der «Feldarbeit» verlassen; dennoch muß man sich vor Augen halten, daß völkerkundliches Feldwissen oft auf recht direktem Wege zur umfassenden Thesen- oder Modellbildung geführt hat (wie z. B. bei den Trobriand-Forschungen BRONISLAW MALINOWSKIS ${ }^{5}$ ) oder aber ausdrücklich mit dem Ziel des Nachweises bzw. der Überprüfung einer theoretischen Position erworben wurde (wie z.B. im Falle der Pygmäenforschungen von Anhängern der urmonotheistischen Theorie ${ }^{6)}$ ).

Selbstverständlich spiegelt sich in diesen - hier nur grob skizzierten - Hauptzielen völkerkundlicher Feldarbeit auch die Geschichte des Faches7); im gleichen $\mathrm{Maße}$, in dem die Ethnologie seit dem Ende des 19. Jahrhunderts an immer mehr Universitäten Eingang fand, hat sie ihr theoretisches Fundament und ihre spezifische Methodik stets weiter ausgebaut - auch wenn im Rückblick der Fortschritt zu großen Teilen nur aus der Erkenntnis von Irrwegen zu bestehen scheint. Immerhin haben sich ungeachtet aller Richtungsunterschiede aus diesem Erfahrungs-, Diskussions- und Reflexionsproze $ß$ mehrere Grunderkenntnisse herausgeschält, die bei der heutigen völkerkundlichen Feldarbeit kaum übergangen werden können und sich in kurzen Worten folgendermaßen formulieren lassen:

1) Auch die traditionell schriftlosen Kulturen, also der zentrale Forschungsgegenstand der Ethnologie, sind hochkomplexe Gebilde mit einer langen eigenen, also jeweils anderen Geschichte - auch wenn diese schwer zu rekonstruieren ist; sie sind weder «einfach» noch «urtümlich». Der moderne Akkulturationsprozeß setzt lediglich den historischen $\mathrm{Ab}$ lauf fort, wenngleich mit noch nie dagewesener Wirkungskraft, die bis zur Auslöschung fast alles Vorausgehenden durchschlagen kann.

2) Jede Kultur stellt eine differenzierte Ordnung sui generis dar; es ist nicht statthaft, Vorstellungen über die funktionale Einbettung irgendeines Einzelphänomens (eines "Kulturelements»), also über seine Position in dieser Ordnung, aus anderen Kulturen zu übertragen - es sei denn zur Überprüfung mit völlig offenem Ausgang, wobei ein Ergebnis erst nach der gründlichen Erarbeitung des gesamten Systems formuliert werden kann.

3) Ohne die Kenntnis dieser (kulturinternen) «Bedeutung» sind Aussagen über die Existenz einzelner Kulturphänomene von beschränktem kulturwissenschaftlichem Wert; immerhin können sie zum Nachweis von historischen Beziehungen dienen, die ihrerseits wiederum den Schlüssel zur Lösung anderer Probleme in der nämlichen Kultur darstellen können.

4) Die sprachliche Dimension ist für das «Verstehen» einer Kultur von besonderem Gewicht - stellt doch, streng genommen, jedes Übersetzen eine Überführung in eine andere, semantisch niemals kongruente Begrifflichkeit und damit bereits eine Interpretation dar. Infolgedessen hat jede Feldarbeit möglichst nahe an der lokalen Originalsprache stattzufinden - oder jedenfalls in einem sprachlichen Medium, das auch in der betreffenden ethnischen Gruppe von vielen fließend gesprochen wird und damit ein gewisses Heimatrecht erlangt hat.

Diese - als anzustrebendes Ideal formulierten - Anforderungen an die ethnographische Feldarbeit lassen leicht erkennen, daß das Durchmessen größerer Gebiete, die von einer Vielzahl sprachlich-kulturell verschiedener Gruppen bewohnt werden, als Expeditionsstil im wesentlichen ausgedient hat und nur noch zur Rekognoszierung oder im Hinblick auf spezielle historische Fragestellungen zumal im Bereich der Sachgüter eine gewisse Berechtigung besitzt. Andererseits ist man mit dem Herantragen einer bestimmten ausformulierten Problemstellung bei der Untersuchung einer noch wenig bekannten ethnischen Gruppe vorsichtig geworden, weil sich damit zugleich Strukturierungen und Gewichtungen einstellen können, die ein Verbiegen des tatsächlich Gegebenen auf Grund vorgefaßter, aber unzutreffender Meinungen bedeuten. Was also bleibt, ist eine sehr offene Fragestellung durch ein fast nur als Schlagwort gegebenes Thema («Ernährung», "Verwandtschaftsordnung» usw.), das ein freies Sich-hinein-arbeiten in den damit bezeichneten, nach Schwerpunkten, Grenzen und Kategorien noch durchaus unklaren Komplex gestattet.

Ferner wird aus den angeführten Überlegungen deutlich, daß ethnographische Feldarbeit heutzutage fast ausschließlich stationär geschieht, d.h. den langfristigen Aufenthalt des Ethnologen innerhalb der gleichen ethnischen Gruppe voraussetzt; konkret bedeutet das eine Zeitspanne von mindestens sechs Monaten (durchschnittlich einem Jahr) in derselben Siedlungseinheit, wobei darunter - im Hinblick auf die personelle Überschaubarkeit - im allgemeinen ein Dorf oder ein Weilerkomplex verstanden wird, dessen Bewohnerzahl mehrere hundert Menschen nicht übersteigen sollte. Diese lokale Eindringlichkeit könnte an sich dadurch verstärkt werden, daß mehrere Ethnologen zusammen am gleichen Platz arbeiteten und damit auch von der - höchst wünschenswerten - Möglichkeit zu gegenseitiger Ergebniskontrolle profitieren könnten; doch hat sich gezeigt, daß auch vergleichsweise große Dörfer durch die gleichzeitige Anwesenheit mehrerer Europäer in verschiedener Hinsicht zu stark belastet werden, vor allem dadurch, daß die wenngleich unbeabsichtigte - Einwirkung der Ethnologen auf das Dorfleben, also die Veränderung des Erkenntnisgegenstandes durch den ohne Subjekt nicht vorstellbaren Akt des Erkennens, das gerade noch tragbare $\mathrm{Ma} ß$ zu überschreiten beginnt, wenn mehr als zwei Personen gleichzeitig anwesend sind und, ob sie es wollen oder nicht, Aufmerksam- 
keit auf sich lenken. Auch das Verhältnis einer im gleichen Dorf arbeitenden größeren Zahl von Ethnologen zueinander ist, wie vielfache Erfahrung zeigt, zunehmenden Spannungen unterworfen, die - von allem Persönlich-Psychischen in solch isolierter Situation einmal abgesehen - u.a. in der begrenzten Zahl geeigneter einheimischer Gesprächspartner ihre Ursache haben können. Auswege aus dieser - durch einander widersprechende Forderungen gekennzeichneten - Situation liegen vor allem darin, entweder in einer größeren Ethnologengruppe in mehreren Dörfern des gleichen Kulturbzw. "Stammes»- oder Sprachgebietes aufgeteilt zu arbeiten, so daß sich die Einzelforschungen gegenseitig ergänzen, oder aber in Form von "restudies» wiederholt in das gleiche Dorf zurückzukehren, um die nötige Breite und Tiefe des Datenmaterials zu erhalten. Allerdings muß man sich darüber im klaren sein, daß auch solche Feldforschungsmodelle Gefahrenquellen enthalten: dorfspezifische Differenzen können weit bedeutsamer sein als erwartet, und das gleiche Dorf kann sich innerhalb weniger Jahre kulturell so stark verändert haben, daß praktisch nur dieser Wandel selbst bzw. sein Niederschlag in einem engeren Lebenssektor das Forschungsthema sein können. Und noch ein letztes ergibt sich aus dem oben (Ziffer 4) Formulierten: nämlich, dass nie auf eine längere Vorbereitungszeit verzichtet werden kann, weil das Erlernen der Lokalsprache - wie weit man immer damit kommen mag - ein mühseliger Prozeß ist, nicht nur wegen der oft beklagenswerten Bescheidenheit des zur Verfügung stehenden Sprachmaterials, sondern auch wegen der besonderen Schwierigkeit eines wenigstens passiven Vertrautwerdens mit nicht-europäischen Sprachen.

Vor dem Hintergrund dieser allgemeineren Überlegungen nun wurde die Mittelsepik-Expedition des Ethnologischen Seminars der Universität Basel geplant und von 1972 bis 1974 mit unterschiedlich langen Feldarbeitsperioden durchgeführt. Im größeren forschungsgeschichtlichen Überblick und mit einer gewissen Dehnung des Begriffs könnte man dieses Projekt zu den «restudies» rechnen - war es doch nicht das erste Mal, daß Basler Ethnologen im Sepik-Gebiet des nördlichen Neuguinea (heute: in der «East Sepik Province» des seit 1975 souveränen Staates Papua New Guinea) tätig waren. So läßt sich, um eine erste Planungsfrage zu beantworten, die Auswahl des Ortes für dieses Projekt verstehen als das Ergebnis eines immer stärkeren räumlichen Eingrenzungsprozesses, der im weiteren Melanesien mit den Forschungen von FRITZ SARASIN auf NeuKaledonien 8) und von FELIX SPEISER auf den Neuen Hebriden9) 1910-1912 begann, durch PAUL WIRZ seit 1915 eine stärkere Konzentration auf Neuguinea (vornehmlich das damalige Holländisch-Neuguinea, das heutige West-Irian ${ }^{10}$ ) erfuhr und im
Jahre 1930, wiederum durch FELIX SPEISER, erstmals (neben den nördlichen Salomonen, Neu-Irland und Neu-Britannien) auch das mittlere SepikGebiet als engeres Arbeitsfeld einschloß11). Seit 1949 reiste dann PAUL WIRZ wiederum vornehmlich im nordöstlichen Neuguinea, wo er auch 1955 starb; nach ihm widmete sich ALFRED BÜHLER, der schon 1931/32 auf Neu-Irland, Neu-Britannien und den Admiralitäts-Inseln gearbeitet hatte, mit besonderem Nachdruck der Sepik-Region, in der er 1955/56 und 1959 große Museumsreisen durchführte ${ }^{12)}$. 1965-1967 arbeitete dann der Schreibende, der schon 1961 zusammen mit EIKE HABERLAND das Flußgebiet des mittleren Sepik und das seitlich angrenzende Hinterland auf einer Sammelreise des Frankfurter Völkerkunde-Museums aufgesucht hatte13), gemeinsam mit CHRISTIAN KAUFMANN und GISELA SCHUSTER in verschiedenen Regionen des Sepik-Beckens bis hinauf ins Quellgebiet, ein noch von CARL AUGUST SCHMITZ vorbereitetes, infolge seines Weggangs nach Frankfurt aber verwaistes Basler Projekt in veränderter Form durchführend ${ }^{14)}$. Diese vom Schweizerischen $\mathrm{Na}$ tionalfonds unterstützte Unternehmung hatte die vertiefte Dokumentation der Basler SepikSammlungen von den verschiedenen, oben erwähnten früheren Sepik-Expeditionen zum Ziel; das bedeutete konkret, daß einerseits anhand mitgenommener fotografischer Abbildungen der betreffenden, nach Tausenden zählenden Museumsgegenstände in einer größeren Zahl von Sepikdörfern Daten zu den technologischen und funktionalen Aspekten dieser Objekte aufgenommen und andererseits in den noch weniger bekannten NebenflußRegionen der dortige Sachgüterbestand durch Erforschung und Erwerb neu dokumentiert wurden. Trotz des vom Expeditionszweck her gegebenen geographisch weiten Ausgreifens bildeten sich auf dieser Reise zwei Schwerpunkte deutlich heraus: einerseits das Kwoma-(Washkuk-)Gebiet, in dem CHRISTIAN KAUFMANN die Töpferei eingehend untersuchte ${ }^{15)}$, und zum andern das im südlichen Hinterland gelegene Dorf Aibom, wo sich die beiden anderen Teilnehmer verschiedenen Themen aus Technologie und Religion zuwandten ${ }^{16}$ ). Bereits damals entstand bei uns der Gedanke, diese beiden Regionen noch einmal und mit mehr Muße aufzusuchen, um zahlreichen Problemen, die neu aufgetaucht oder in der vergleichsweise kurzen Zeit nicht lösbar waren, weiter nachzugehen.

Dieser knappe forschungsgeschichtliche Abriss sollte zeigen, daß die Frage der Ortswahl für eine Feldarbeit in unserem Beispiel mit fast zwingender Logik von den vorausgegangenen Expeditionen her zu beantworten war: es galt, Begonnenes vertiefend weiterzuführen und damit auch die Vorteile, die sich aus jahrzehntelangen Beziehungen zu einer bestimmten Region (einschließlich der Publikationen, 
des Bestandes an Kulturdokumenten, der Feldnotizen, des persönlichen Wissens usw.) ergaben, nicht $\mathrm{zu}$ verschenken. Insofern fiel die Entscheidung über den Ort der Feldarbeit primär von der Situation in Basel aus; doch war andererseits das mittlere Sepik-Gebiet seit den Forschungen von GREGORY BATESON ${ }^{17)}$ in den dreißiger Jahren nicht mehr Gegenstand eingehender Feldarbeit, so daß sich hier die günstige Gelegenheit bot, auf dem durch eine große frühere Leistung schon Bekannten weiter aufzubauen, bestimmte thematische Komplexe die damals eher am Rande blieben - zusätzlich zu erforschen und (jetzt im engeren Sinn eines «restudy») den damaligen Befund mit dem heutigen Bild zu vergleichen. Die Basler Feldarbeit von 1972 bis 1974 kann also nicht beanspruchen, in geographischem Sinne in unbekannte Räume vorgedrungen zu sein und neue "Völker» oder Kulturen entdeckt zu haben: die ersten weißen Kontakte mit dem mittleren Sepik-Gebiet datieren aus den achtziger Jahren des vorigen Jahrhunderts, ausführlichere Berichte liegen noch aus der bis zum Ersten Weltkrieg dauernden deutschen Kolonialzeit vor ${ }^{18)}$, und neben der australischen Kolonialverwaltung war die katholische Mission (SVD) seit Jahrzehnten im mittleren und unteren Sepik-Gebiet sowie im beiderseitigen Hinterland tätig. Auch solche Gebiete also, in denen «unberührte» Kulturzustände kaum noch zu erwarten sind und $z$. B. früher so zentrale Kulturphänomene wie Krieg, Kopfjagd und Kannibalismus von den europäischen Administrationen mit Gewalt und Erfolg unterbunden wurden, sind heute durchaus angemessene Räume für die ethnologische Feldforschung - nicht nur deshalb, weil unberührte Orte auf der Welt immer rarer geworden sind, sondern auch, weil sie eine andere Art der Feldarbeit ermöglichen. Denn während bei solchen ethnischen Gruppen (z. B. im amazonischen Regenwaldgebiet oder auch in abgelegeneren Teilen Neuguineas), mit denen praktisch zum ersten Mal Kontakt aufgenommen wird, die Erfassung kultureller Daten sich auf Grund der schwierigen Sprachsituation vorwiegend auf das sichtbare Kulturbild konzentriert, sind in den schon länger akkulturierten Gebieten einerseits Gespräche - und damit das Eindringen in den so viel größeren nicht sichtbaren Kulturbereich - schon aus rein sprachlichen Gründen leichter möglich, andererseits bei den einheimischen Bevölkerungen mehr Anlaß und Bereitschaft vorhanden, die traditionellen eigenen Lebensformen nicht mehr als selbstverständlich zu werten, sondern ihnen nachdenklich gegenüberzustehen und damit die Arbeit des europäischen Forschers teils zu erleichtern, teils durch eine bereichernde neue Dimension zugleich zu komplizieren. So zählen nach heutigem allgemeinem Verständnis in unserem Fach Kulturräume von sehr unterschiedlichem Akkulturationsniveau, also von verschieden starker weißer Überfremdung, in gleicher Weise zu den angemessenen ethnologischen Feldarbeitsgebieten; das geschilderte Basler Beispiel, die Mittelsepik-Region in Papua New Guinea, mag etwa in der Mitte dieser Spannweite liegen, die von der romantischen ersten nennenswerten Erforschung einer vielleicht nur dem Namen nach bekannten ethnischen Gruppe bis hin zur UrbanEthnologie reicht, also dem Studium der Lebensformen ethnischer Gruppen (oft: Minderheiten) unter städtischen Lebensbedingungen in der Dritten Welt. Die Grenze der ethnologischen Feldarbeit wird naheliegenderweise dort erreicht, wo ethnische Abgrenzungen selbst nicht mehr gesehen, ethnische Identitäten nicht mehr erlebt werden, seien sie nun vorwiegend sprachlicher, allgemein-kultureller oder historisch-regionaler Art. So bietet sich nach wie vor für die ethnologische Feldarbeit eine Vielfalt von Möglichkeiten, die weit eher von politischen als von wissenschaftlichen Faktoren beschränkt werden und trotz der beträchtlichen $\mathrm{Zu}$ nahme der Ethnologen in vielen Ländern so zahlreich sind, daß viele ungenutzt bleiben. Nicht immer ist, wie im Falle dieses Basler Projektes, eine Traditionslinie weiterzuführen, die ihrerseits ja auch einmal mit einer erstmaligen Entscheidung für eine bestimmte Erdregion (hier: Melanesien) begann; in vielen Fällen werden - auf Grund unterschiedlicher Überlegungen, denen wir uns jetzt zuwenden wollen - gerade solche Räume ausgesucht, in denen noch keine gründlichere Feldarbeit geleistet worden ist.

Denn selbstverständlich ist die Ortswahl unmittelbar mit der wissenschaftlichen Fragestellung verbunden - nicht nur im Hinblick auf das gerade erörterte Problem des Akkulturationsgrades einer Region, sondern auch, und vor allem, hinsichtlich der Existenz bzw. Differenziertheit eines am hiesigen Schreibtisch interessant erscheinenden Kulturphänomens. So lassen sich grundsätzlich zwei Wege der Planung und Durchführung ethnologischer Feldarbeit unterscheiden, die beide wissenschaftlich gleichermaßen gerechtfertigt sind. Die eine Überlegung geht regional bzw. lokal vor, indem sie sich primär einer bestimmten Region, einer bestimmten ethnischen Gruppe, einem bestimmten Dorf zuwendet und hier in einem vom jeweiligen Forschungsstand abhängigen Maße allgemeinere oder detailliertere ethnologische Feldarbeit leistet; je nach dem vorhandenen Arbeitspotential (Zahl der Mitarbeiter, Länge des Aufenthaltes, Umfang der Mittel usw.) und der allgemeinen Orientierung der betreffenden Forscher innerhalb des Fachs werden sich dann in einer sekundären Überlegung bestimmte Themen als vordringliche Forschungsaufgaben herauskristallisieren. Der zweite Weg geht primär von einem als wissenschaftlich interessant erkannten Thema aus und bestimmt von da aus 
nach dem gegebenen, leider oft veralteten Wissensstand den Ort der Feldarbeit - wobei sich im einen wie im anderen Falle nach aller Erfahrung noch beträchtliche Modifizierungen am Arbeitsort selbst zu ergeben pflegen, da die konkreten aktuellen Verhältnisse, zumal in personeller Hinsicht, normalerweise ja nicht zuverlässig bekannt sind.

Das Basler Neuguinea-Projekt 1972-74 war, wie sich aus früher Gesagtem schon ableiten läßt, eine Unternehmung der erstgenannten Art: Ausgangspunkt war der Wunsch, über die Kultur der Iatmul - wie seit GREGORY BATESON ${ }^{17)}$ die Bewohner der Mittelsepik-Dörfer genannt werden - möglichst viel zu erfahren. Aus dem, was wir auf der Expedition 1965/67 gehört und gesehen hatten bzw. in der vorliegenden Literatur lesen konnten, war klar, daß nicht ein einzelner oder zwei, sondern nur eine Gruppe von mehreren Ethnologen in der Lage sein könnte, mit diesem "holistischen» Ansatz die Kultur der Iatmul in sinnvoller Weise weiter zu erforschen; so wurde unter der Leitung des Schreibenden eine Gruppe jüngerer Ethnologen aus dem Basler Seminar gebildet, die folgende Mitglieder umfaßte: BRIGITTA und JÖRG HAUSER-SCHÄUBLIN, MARKUS SCHINDLBECK, JURG SCHMID, GISELA und MEINHARD SCHUSTER, MILAN STANEK, JÜRG WASSMANN und FLORENCE WEISS. Parallel dazu führte, ebenfalls vom Schweizerischen Nationalfonds unterstützt, mit nur geringer zeitlicher Verschiebung auch CHRISTIAN KAUFMANN vom Basler Völkerkunde-Museum seine frühere Feldarbeit bei den Kwoma im Washkuk-Gebiet, jetzt zusammen mit ANNEMARIE KAUFMANN-HEINIMANN, mit dem Ziel der weiteren Erforschung der Kwoma-Kultur insbesondere in religions- und kunstethnologischer sowie ethnotechnologischer Richtung weiter ${ }^{19)}$.

Selbstverständlich mußten für die größere Gruppe jetzt weitere Einteilungen getroffen werden, denn gemäß dem eingangs in allgemeinerer Form Ausgeführten war weder der Idealfall möglich, daß alle Expeditionsmitglieder im gleichen Dorfe an verschiedenen Themen arbeiteten, noch war es - angesichts der kulturellen und sprachlichen Gleichheit bzw. Ähnlichkeit der einzelnen Sepik-Dörfer sinnvoll und notwendig, daß alle in verschiedenen Dörfern das gleiche Thema untersuchten. Es sei gern eingeräumt, daß auch dieser Forschungsansatz methodisch sehr verlockend gewesen wäre also z. B. in sechs verschiedenen Dörfern, die nach allgemeinen ethnologischen Standards als der gleichen Kultur zugehörig gelten müssen, die Rolle der Frau oder die Nahrungsbeschaffung zu untersuchen; jedoch hätte dies erstens bedeutet, daß in allen Dörfern wesentliche andere, für die genannten Themen als Bezugsfelder bedeutungsvolle Kulturbereiche unerforscht geblieben wären, und zweitens, daß kleineren Unterschieden zwischen den Dörfern ein überproportionales Gewicht zugekom- men wäre, der Forschungsgewinn im ganzen und zumal im Hinblick auf unsere Kenntnis des SepikGebietes sich also in unnötig engen Grenzen gehalten hätte. So entschlossen wir uns dazu, daß zwar von verschiedenen Teilnehmern in verschiedenen Mittelsepikdörfern verschiedene Themen zu bearbeiten seien, den individuellen wissenschaftlichen Neigungen entsprechend, andererseits aber erstens die Abstände zwischen den auszuwählenden Dörfern so gering sein sollten, daß der gegenseitige Kontakt durch Einzelbesuch und Gruppenbesprechung leicht möglich wäre, und zweitens die Dorf-, Clan- und Hälftenorganisation als allgemeiner, den Vergleich erleichternder und die Einzelthemen überwölbender Bezugsraster von allen Teilnehmern aufgenommen werden sollte.

So arbeiteten BRIGITTA HAUSER-SCHÄUBLIN ${ }^{1)}$ in Kararau, unterstützt von JÖRG HAUSER-SCHÄUBLIN, der als Nicht-Ethnologe auf eigene Rechnung teilnahm, über die Rolle der Frau, MARKUS SCHINDLBECK in Gaikorobi über den Sago, also die Hauptnahrungspflanze, in allen ihren Zusammenhängen, JÜRG SCHMID in Yentshan über das Initiationswesen und die Migration, Milan Stanek in Palimbei über die sozialen Aspekte der Mythologie, JÜRG WASSMANN in Kandingei über die Beziehungen zwischen den kultischen Gesängen und der Sozialorganisation, FLORENCE WEISS ebenfalls in Palimbei über die Lebenswelt des. Kindes, schließlich meine Frau und ich an unserem schon von früher her bekannten Platz Aibom über die Sozialorganisation in ihren mythologischen und räumlichen Aspekten ${ }^{1)}$. Diese thematische Grobgliederung, die - wie früher erörtert - statt einzelner Fragestellungen nur die Blickrichtung angibt, ist, wie leicht erkenntlich, teils an den spezifischen Bedingungen des Mittelsepik orientiert («Sago», "Kultgesänge»), teils eher allgemein gehalten wie z. B. die beiden Komplexe «Frau» und "Kind», die sich für die beiden genannten Ethnologinnen besonders empfahlen und eben auch nur von Frauen befriedigend erforscht werden können. In allen Fällen wurden innerhalb der Themenblöcke auch gemäß den örtlichen Möglichkeiten noch besondere Schwerpunkte gesetzt, so daß die Überlappungen nicht störend ins Gewicht fielen oder sogar als Vergleichsdaten aus einem anderen Dorf nützlich waren. In einem Fall erwies sich gegenüber dem ursprünglichen Plan vom Thema und den Arbeitsmöglichkeiten her ein Dorfwechsel als notwendig. Hinsichtlich der ethnischen Streuung ist anzufügen, daß in allen drei Teilgruppen der Iatmul gearbeitet wurde (Kandingei = West-, Palimbei und Yentshan = Zentral-, Kararau $=$ Ost-Iatmul ${ }^{20)}$; andererseits sind zwei der genannten Dörfer nur mit Vorbehalten der IatmulKultur zuzurechnen. Gaikorobi im nördlichen Hinterland gehört zum Gebiet der Sawos, die einen dem Iatmul verwandten Dialekt der Ndu- 


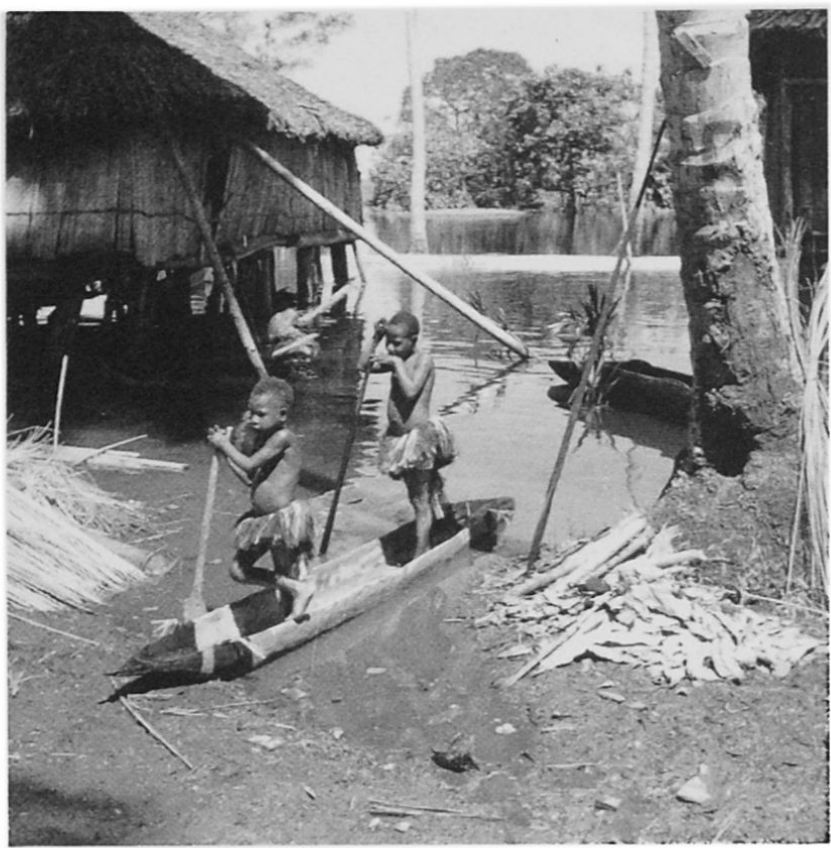

Abb. 1: Zwei Mädchen aus dem Dorfe Aibom im südlichen Hinterland des Mittelsepik (Papua New Guinea) in ihrem neu angefertigten bemalten Einbaum. Der sonst trockene Dorfboden ist vom regenzeitlichen Hochwasser weithin überschwemmt. Der traditionelle Frauenschurz wird heute nur noch zu festlichen Anlässen getragen.

Sprachfamilie sprechen und die Hauptsagolieferanten der Flußdörfer sind, sich aber kulturell in manchem von ihnen abheben; Aibom, auf der Südseite des Sepik an einem kleinen Nebenfluß und nahe dem Chambri-See landeinwärts gelegen, bildete früher zusammen mit Chambri, soweit sich das heute beurteilen läßt, eine sprachlich-kulturelle Einheit und wurde erst vor wenigen Generationen von der Zentral-Iatmul-Kultur überfremdet.

Die Arbeitsdauer der Expeditionsmitglieder schwankte je nach den persönlich-beruflichen Bedingungen zwischen 6 und 18 Monaten innerhalb des Zeitraums von August 1972 bis April 1974; vorausgegangen war - neben der selbstverständlichen längeren Beschäftigung mit der Ethnologie Neuguineas im allgemeinen und der des Sepik-Gebietes im besonderen - eine einjährige sprachliche Vorbereitungszeit, in der der Schreibende einen Pidgin-Kurs abhielt und wir uns gemeinsam auf der Basis von Tonbändern und schriftlich niedergelegtem Sprachmaterial, das wir zum Teil und auf unseren Wunsch hin von P.Z'GRAGGEN SVD erhielten, die IatmulSprache anzueignen versuchten.

Blicken wir jetzt auf die eingangs genannten drei größeren Zielsetzungen völkerkundlicher Feldforschung zurück, so ist zu sagen, daß das Basler Neuguinea-Projekt 1972-74 deutlich dem an zweiter Stelle genannten «monographischen» Forschungsansatz zugehört. Die sechs MittelsepikDörfer, in denen wir tätig waren, liegen mit weni-

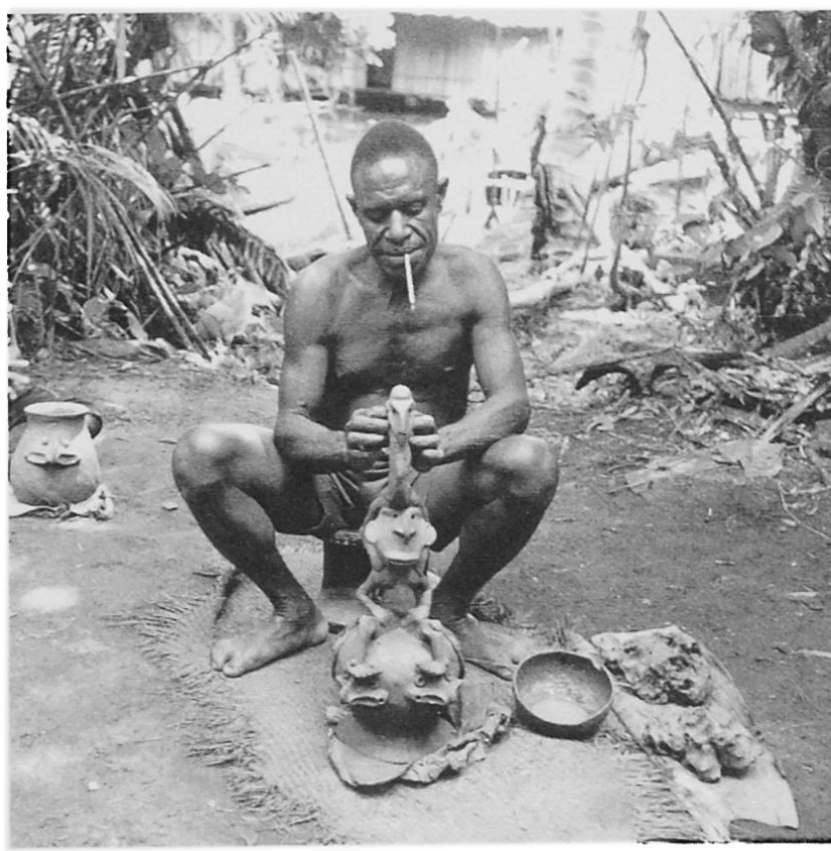

Abb. 2: Mbouindu aus dem Töpferdorf Aibom im südlichen Hinterland des Mittelsepik (Papua New Guinea) beim Herstellen eines Dachaufsatzes. Diese figürlichen Tonplastiken wurden früher auf den Firstspitzen von Kulthäusern angebracht; die Darstellung nimmt auf die Mythologie Bezug. Heute stellt man solche Tonwaren, oft in kaum verminderter künstlerischer Qualität, zum Verkauf her.

gen Stunden Abstand, also kleinräumig, beieinander, und die ethnographischen Daten wurden, jeweils von einem thematischen Zentrum ausgehend, mit besonderer Bemühung um ihre kulturspezifischen gegenseitigen Beziehungen aufgenommen, so daß aus der Erfassung der Zusammenhänge der «Sinn» der Einzelphänomene deutlich werden und im ganzen, soweit möglich, ein integriertes Kulturbild entstehen soll, wenn einmal alles Feldmaterial aufgearbeitet sein wird. Unser Ziel war denn auch nicht, die Iatmul-Kultur als Beleg für einen wie auch immer definierten Kulturtyp darzustellen oder an ihrem Beispiel eine bestimmte kulturwissenschaftliche Einzelhypothese zu erweisen, sondern vielmehr, durch ein möglichst vielseitiges Eindringen und unter breiter Verwertung sprachlicher Zeugnisse das geistige System zu erfassen, als das die Iatmul-Kultur im Denken jedes einzelnen Iatmul existiert, in den Texten oder anderen Formen (z. B. Kunstwerken) vor uns hintritt und im Dorfleben das Handeln steuert. Die Iatmul-Kultur wurde final um ihrer selbst willen, nicht instrumental im Dienste eines von außen herangetragenen Gedankens studiert - sofern man nicht den Wunsch zum «Verstehen» seitens des Außenstehenden schon als einen solchen betrachtet.

Allerdings war noch eine weitere Überlegung an zentraler Stelle im Spiel - nämlich die, daß es grundsätzlich und in jedem einzelnen Falle von unbezweifelbarem Wert sei, fremde Kulturen, die 
nicht oder nur in sehr begrenztem Maße über sich selbst reflektieren, sich also nicht selbst zum Erkenntnisgegenstand machen und auch nicht über unsere verfeinerten Dokumentationstechniken verfügen, als einmalige, unwiederbringliche Gestaltungen des Menschengeistes in ihrer Besonderheit zu erforschen und für die Mit- und Nachwelt, soweit als möglich, in Berichtform bereitzustellen bzw. zu bewahren. $\mathrm{Zu}$ den Interessenten an solchen Darstellungen zählen mittlerweile jedoch nicht mehr nur «europäische» Leser, sondern auch diejenigen selbst, von denen diese völkerkundlichen Berichte handeln; denn anders als noch vor wenigen Jahrzehnten ist jetzt zumal in den stärker akkulturierten Gebieten die junge Generation zunehmend in der Lage, auf Grund der weiter verbreiteten Schulbildung europäische Sprachen zu lesen - in unserem Falle das Englische. Damit entstehen einerseits Übersetzungsprobleme, denn neben dem Iatmul, das bis jetzt noch nicht als geschriebene Sprache etabliert ist, kommen grundsätzlich drei verschiedene sprachliche Ebenen in Frage: das Pidgin (als die von praktisch allen Dorfbewohnern des Mittelsepik und z. B. auch im neuguinensischen Radio gesprochene Verkehrssprache), das Englische (als Wissenschaftssprache an der Landesuniversität in Port Moresby bzw. als die in Neuguinea übliche "Weltsprache») oder das Deutsche (als Muttersprache der Autoren, in unserem Fall). Andererseits tauchen auch ethische Probleme auf, die früher unbekannt waren bzw. übersehen werden konnten denn wie sollen wir mit Daten verfahren, die sich auf Konflikte in der persönlich-dörflichen Sphäre beziehen, wie mit Angaben, die für das Kulturverständnis wichtig, aber partiell geheim sind und deshalb nur vertraulich mitgeteilt wurden, oder mit Informationen, die das Verhältnis zwischen Dorfbewohnern und Verwaltung bzw. Regierung betreffen und deshalb unter Umständen von dieser zum Nachteil jener benutzt werden könnten? Man wird sich hier wohl immer zugunsten des ethisch Verantwortbaren und zuungunsten einer rigorosen Wissenschaftlichkeit zu entscheiden haben - und damit wiederum den nachfolgenden Generationen am Sepik, für die ja die Ethnologie die Geschichte ihrer Vorfahren schreibt, einen Teil des an sich möglichen Wissens vorenthalten.

Aus diesen Erwägungen ist ferner deutlich geworden, daß für die ethnologische Feldarbeit heute neben der traditionellen Zulieferung an die nordatlantisch geprägte Wissenschaftswelt auch der unmittelbare und spezifische Rückfluß wissenschaftlicher Ergebnisse an die Forschungsländer selbst als wesentliche Aufgabe hinzugekommen ist; in manchen Staaten der Dritten Welt ist die Mitnahme einheimischer Ethnologen auf Feldforschungsprojekte vorgeschrieben, während in Papua New Guinea z. Zt. die Zusammenarbeit mit einheimischen kul- turwissenschaftlichen Institutionen (Department of Anthropology der Universität, Institute of Papua New Guinea Studies, National Museum) erwartet und von uns selbstverständlich gerne geleistet wird. Im besonderen wurde gemeinsam mit dem Basler Museum für Völkerkunde und mit Unterstützung durch den Schweizerischen Nationalfonds jetzt unter der Leitung von CHRISTIAN KAUFMANN ein Programm in Angriff genommen, das die wissenschaftliche Auswertung aller auf den verschiedenen Basler Expeditionen erworbenen Kenntnisse zu Basler Museumsobjekten vom Sepik einschließlich ihrer Publikation auf Microfiches und ihres Austausches mit gleichartig aufbereitetem Material aus dem $\mathrm{Mu}$ see de l'homme in Paris und dem National Museum in Port Moresby zum Ziel hat. Daneben ist schon seit längerem im Ethnologischen Seminar ein auf der Auswertung der ethnographischen Literatur bzw. der Reise- oder Verwaltungsberichte seit den 1870er Jahren beruhender «Ethnographisch-Historischer Atlas von Papua New Guinea» in Arbeit, der auf zeitlich gestuften Regionalkarten die in den vergangenen hundert Jahren gemeldeten Positionen von Sprachgruppen und ethnischen Einheiten enthält und damit einerseits die Erforschungsgeschichte, andererseits die Bevölkerungsbewegungen wiedergibt. Der erste, das festländische Papua New Guinea betreffende Teil ist fertiggestellt und soll demnächst in Zusammenarbeit mit dem National Cultural Council von Papua New Guinea publiziert werden. Schließlich ist noch auf zwei besondere Sparten wissenschaftlicher Dokumentation zu verweisen, in denen eine Rückleitung von Ergebnissen der Feldarbeit an das Land, wo sie geschah, erfolgt: Film und Ton. Auf verschiedenen Expeditionen haben wir zum Teil selbst, zuletzt auch in $\mathrm{Zu}$ sammenarbeit mit HERMANN SCHLENKER, Schwenningen, in mehreren Dörfern des Mittelsepik Schmalfilme (schwarzweiß und farbig, $8 \mathrm{~mm}$ und $16 \mathrm{~mm}$, mit und ohne Ton) zu verschiedenen Themen, vornehmlich aus dem handwerklichen und kultischen Bereich gedreht und dann im Institut für den Wissenschaftlichen Film in Göttingen bearbeitet ${ }^{21)}$; Kopien einiger Filme wurden dem National Museum in Port Moresby bereits übersandt, zusammen mit den wissenschaftlichen Begleitpublikationen. Ferner wurden Tonbandaufnahmen, die der Regierungsarzt BOB MACLENNAN während mehrerer Jahre im mittleren Sepik-Gebiet von einheimischer Instrumentalmusik anfertigte, gemeinsam mit GORDON F. SPEARRITT, Musikwissenschaftler an der Universität Brisbane, bei Gelegenheit zweier mehrmonatiger Gastaufenthalte im Ethnologischen Seminar in Basel bearbeitet ${ }^{22)}$; aus diesen Aufnahmen werden jetzt zusammen mit dem Institut für Musikwissenschaft der Universität Basel ${ }^{23)}$, dem Institute of Papua New Guinea Studies in Port Moresby und dem Bärenreiter-Verlag zwei Schallplatten mit aus- 
führlich dokumentierter einheimischer Musik hergestellt.

Neben der wissenschaftlichen Forschung und der Publikation ihrer Ergebnisse einerseits und dem Problem der Rückleitung dieses neuen Wissens- und Erkenntnisstandes an das Land, in dem die Forschung stattfand, andererseits wird selbstverständlich auch heute - wie schon seit dem Beginn der wissenschaftlichen Völkerkunde - eine wichtige Aufgabe darin gesehen, Wissen über fremde Kulturen auch hierzulande an ein breiteres Publikum weiterzugeben; dies um so mehr dann, wenn es sich um Resultate jüngster Feldforschung handelt, die neben dem traditionellen - also langfristig konstanten - Kulturbild auch seinen aktuellen Wandel berücksichtigen. Unmittelbarkeit, Erfahrungsgehalt und Anschauungsfülle zusammen mit der Vielfalt technischer Dokumentationsmittel (Foto, Film, Tonband) bewirken, daß der konkrete Feldforschungsbericht im allgemeinen auf besonders große Resonanz stößt. Neben solchen Einzel- oder Serienveranstaltungen - die natürlich auch in Basel in verschiedenem Rahmen und verschiedenen Medien stattfanden - bieten sich für eine größere Breitenwirkung noch vor allem zwei Wege an: die Gestaltung von Ausstellungen in Verbindung mit einem Museum oder die Publikation populärwissenschaftlicher Bücher. Während sich für den letzteren Weg in unserem Fall eher zufällig eine Möglichkeit ergab $^{24)}$, ist die Durchführung von mehrmonatigen Wechselausstellungen - die im allgemeinen die Dartbietung von neuestem und regional eng begrenztem Material eher gestatten als Dauerausstellungen - in Basel seit Jahrzehnten Tradition. So wurden alte und neue Sachgüter und Dokumente vom Mittelsepik seit 1965 vor allem in drei Ausstellungen im Basler Museum für Völkerkunde gezeigt: «Farbe, Motiv, Funktion» 1968/69, "Jugend und Gesellschaft»1973/74, «Papua Niugini - ein Inselstaat im Werden» 1974/76 25 , eine Reihe anderer Ausstellungen, die den einheimischen Kulturen am Sepik im Titel gewidmet waren oder Kulturgüter aus diesem Raum in anderem Zusammenhang zeigten, waren in den $50 \mathrm{er}$ oder früheren 60er Jahren vorausgegangen, vor allem im Zusammenhang mit den Expeditionen von F. SPEISER, P. WIRZ und A. BÜHLER (z. B. «Kunstwerke vom Sepik» 1954, «Kunststile am Sepik»1960'6) $)$.

So sind einerseits die Mitglieder der in diesem Beitrag behandelten Basler Neuguinea-Expedition 1972/74 noch mit der weiteren Verwertung und Ausarbeitung ihres Feldmaterials befaßt; zum anderen war diese Forschungsreise jedoch nicht als Ende, sondern nur als weiteres Glied in einer sich fortsetzenden Kette gedacht. BRIGITTA und JÖRG HAUSER-SCHÄUBLIN haben zusammen mit BARBARA GREUB vom Herbst 1978 an ein Jahr bzw. neun Monate, vom Schweizerischen Nationalfonds unter- stützt, im Maprik-Hügelland, das nördlich an die Mittelsepik-Region angrenzt, an mehreren religions- und wirtschaftsethnologischen Themen gearbeitet, die sich zum Teil im Anschluß an die Iatmul-Forschungen aufdrängten, zum Teil als eigenständige Untersuchungskomplexe bei den - mit den Iatmul sprachverwandten - Abelam im Maprik-Gebiet ergaben; im Auftrag des Basler Museums für Völkerkunde wurde dabei auch im besonderen das große Abelam-Kulthaus, von dem ein Exemplar aus Originalteilen im Museum errichtet werden wird, technologisch und ikonographisch erforscht. Ferner ist MARKUS SCHINDLBECK im August 1979 mit Mitteln der Deutschen Forschungsgemeinschaft für anderthalb Jahre in die Region von Dreikikir ausgereist, die im Nordwesten an das mittlere Sepik-Gebiet anschließt; er wird sich dort neben der selbstverständlich unumgänglichen allgemeinen Ethnographie - im besonderen den Problemen von Anbau und Handwerk im gegenwärtigen kulturellen Wandel widmen. Schließlich sind im September 1979 FLORENCE WEISS und MILAN STANEK nach Papua New Guinea zurückgeflogen, um einerseits F.MORGENTHALER, der zusammen mit P. PARIN durch psychoanalytische Forschungen in Westafrika bekannt wurde ${ }^{27)}$, bei ähnlichen Untersuchungen am Mittelsepik zu unterstützen und um andererseits die Möglichkeiten für eine spätere urbanethnologische Feldarbeit in Rabaul zu prüfen. Diese soll sich eingehend mit der für Papua New Guinea besonders gravierenden Frage der Migration von den Binnenland-Dörfern in die Küstenstädte befassen und dabei nicht, wie es oft geschieht, von den Stadtbewohnern ausgehen, sondern auf der 1972-74 erlangten genauen Kenntnis einheimischer Dorfbevölkerungen - in diesem Fall von Palimbei - aufbauen, von denen Teile in die Städte abgewandert sind. Weitere Feldarbeiten im Sepik-Bereich sind für die nächsten Jahre geplant.

\section{Literatur bzw. Anmerkungen}

1) HAUSER-SCHÄUBLIN, B.: Frauen in Kararau. Zur Rolle der Frau bei den Iatmul am Mittelsepik, Papua New Guinea. Basler Beiträge zur Ethnologie, Bd. 18, Basel 1977.

SCHINDLBECK, M.: Sago bei den Sawos (Mittelsepik, Papua New Guinea). Untersuchungen über die Bedeutung von Sago in Wirtschaft, Sozialordnung und Religion. Basler Beiträge zur Ethnologie, Bd. 19, Basel 1980.

WEISS, F.: Kinder schildern ihren Alltag. Untersuchungen zur Teilnahme der Kinder am ökonomischen System der Dorfgemeinschaft von $\mathrm{Pa}$ limbei (Mittelsepik, Papua New Guinea). 
STANEK, M.: Sozialordnung und Mythik in Palimbei. Bausteine zur ganzheitlichen Beschreibung einer Dorfgemeinschaft am Mittelsepik (Papua New Guinea).

WASSMANN, J.: Der Gesang an den Fliegenden Hund. Untersuchungen zu den totemistischen Gesängen und geheimen Namen des Dorfes Kandingei am Mittelsepik (Papua New Guinea).

Die Arbeiten von F.WEISS, J.WASSMANN und M. STANEK werden gleichfalls in der Reihe Basler Beiträge zur Ethnologie erscheinen (voraussichtlich als Nr.20, 21, 22).

2) HERODOT: Historien. Tusculum Bücherei, München 1963.

3) TACITUS, P.C.: Germania. Goldmann-Klassiker Bd. 7518, München.

4) Zugänglich z.B. in den Editionen von LEONHARD SCHULTZE-JENA, (deutsch) und ANDERSON/DIBBLE (englisch).

5) MALINOWSKI, B.: Argonauts of the Western Pacific, London 1922.

MALINOWSKI, B.: A Scientific Theory of Culture, Chapel Hill/North Carolina 1944.

6) Vgl. die Publikationen von P.W.SCHMIDT (z. B.: Der Ursprung der Gottesidee, Münster 19121955) und P.P.SCHEBESTA (z. B.: Die BambutiPygmäen vom Ituri, Brüssel 1938-1948).

7) Vgl. dazu: w.E. MÜhlmanN, Geschichte der Anthropologie, Frankfurt 1968: oder HARRIS, M.: The Rise of Anthropological Theory, London 1969.

8) SARASIN, F.: Ethnologie der Neu-Caledonier und Loyalty-Insulaner, München 1926.

9) SPEISER, F.: Ethnographische Materialien aus den Neuen Hebriden und den Banks-Inseln. Berlin 1923.

10) WIRZ, P.: Die Marind-anim von HolländischSüd-Neuguinea. Hamburg 1922/25.

11) SPEISER, F.: Eine Initiationszeremonie in Kambrambo am Sepik, Neuguinea 1937. In: Ethnologischer Anzeiger 4, 1937, S. 153-157.

12) Vgl. die Publikationen von A.BÜHLER, z. B.: Die Sepikexpedition 1959 des Museums für Völkerkunde zu Basel. In: Regio Basiliensis II, S. 77-97, Basel 1960/61.

Kultkrokodile vom Korewori (Sepik-Distrikt, Territorium Neuguinea). Zeitschr. f. Ethnologie, Bd. 86, Heft 2, S. 183-207. Braunschweig 1961.

Kulturkontakt und Kulturzerfall. In: Acta Tropica 14, S. 1-35, 1957.
13) HABERLAND, E., SCHUSTER, M.: Sepik. Kunst aus Neuguinea. Ausstellungsführer. Frankfurt 1964.

14) SCHUSTER, M.: Vorläufiger Bericht über die Sepik-Expedition 1965-1967 des Museums für Völkerkunde zu Basel. In: Verhandl. Naturf. Ges. Basel, Band 78, Nr. 1, S. 268-282, 1967.

15) KAUfManN, C.: Das Töpferhandwerk der Kwoma in Nord-Neuguinea. Beiträge zur Systematik primärer Töpfereiverfahren. Basler Beiträge zur Ethnologie, Bd. 12. Basel 1972.

16) SCHUSTER, M.: Die Töpfergottheit von Aibom. In: Paideum, Bd. XV, S. 140-159, 1969.

SCHUSTER, M.: Zum Verständnis religiöser Phänomene der Naturvölker. In: Verhandl. Naturf. Ges. Basel, Bd. 80, Nr.2, S. 226-236, 1970.

SCHUSTER, G.und M.: Begleitpublikationen zu ethnotechnologischen Filmen aus Aibom, publiziert beim Institut für den Wissenschaftlichen Film, Göttingen.

17) BATESON, G.: Social Structure of the Iatmul People of the Sepik River. In: Oceania II, S. 245291; 401-451. 1932.

BATESON, G.: Naven. 1936. $2^{\text {nd }}$ ed. Stanford 1958.

18) Z. B.: O. RECHE, Der Kaiserin-Augusta-Fluß. Hamburg 1913.

W. BEHRMANN, Im Stromgebiet des Sepik. Berlin 1922.

19) KAUFMANN, C.: Begleitpublikationen zu kunstethnologischen Filmen von den Kwoma, z.Zt. im Druck beim Institut für den Wissenschaftlichen Film, Göttingen.

KAUFMANN, C.: Art and Artists in the Context of Kwoma Society. In: Mead, S. M. (Ed.), Exploring the Visual Art of Oceania, S. 310-334, Honolulu 1979.

20) Vgl. M.SCHUSTER, Zur Dorfgeschichte von Soatmeli. In: Festschrift Helmut Petri, S. 475-491, Köln 1973.

21) Vgl. auch Anm. 16; die Begleitpublikationen zu den späteren Filmen sind z. Zt. noch in Bearbeitung bzw. im Druck.

22) Vgl. G.D.SPEARritT, Music of the Middle Sepik River (New Guinea) - a preliminary survey. In: Studies in Music, Nr.8, S. 101-109, 1974. Eine ausführliche Bearbeitung der Musik vom Mittelsepik durch den gleichen Verfasser steht kurz vor dem Abschluß.

23) Prof. H. OESCH sei an dieser Stelle für seine kompetente, tatkräftige Unterstützung, ohne die dieser Plan nicht zu verwirklichen wäre, auf das herzlichste gedankt. 
24) HARRER, H.: Unter Papuas. Mit Beiträgen von C. KAUFMANN, G. SCHUSTER, M. STANEK, F. WEISS. Graz 1976.

25) SCHUSTER, M.: Farbe-Motiv-Funktion. Zur Malerei von Naturvölkern. Museum für Völkerkunde Basel, 1968.

HAUSER-SCHÄUBLIN, B.: Bei den Iatmul: Aufwachsen in einer zweigeteilten Kultur. In: Jugend und Gesellschaft. Museum für Völkerkunde Basel, 1973, S. 33-35.

KAUFMANN, C.: Papua Niugini. Ein Inselstaat im Werden. Museum für Völkerkunde Basel, 1975.

26) Vgl. die gleichnamigen Kataloge von P. WIRZ bzw. A. BUUHLER, Basel; siehe auch den Beitrag von C. KAUFMANN in diesem Heft.

27) PARIN, P./MORGENTHALER, F./PARIN-MATTHEY, G.: Die Weißen denken zuviel. Kindler Taschenbücher 2079. München. 